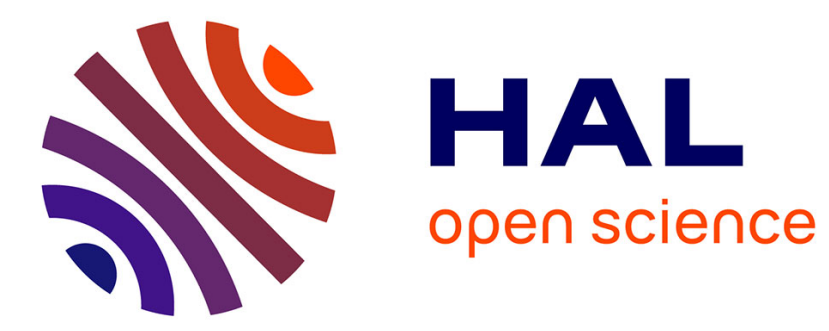

\title{
Les propriétés acoustiques des verres isolants à basse température
}

\author{
P. Doussineau
}

\section{To cite this version:}

P. Doussineau. Les propriétés acoustiques des verres isolants à basse température. Revue de Physique Appliquée, 1977, 12 (5), pp.809-816. 10.1051/rphysap:01977001205080900 . jpa-00244246

\section{HAL Id: jpa-00244246 https://hal.science/jpa-00244246}

Submitted on 1 Jan 1977

HAL is a multi-disciplinary open access archive for the deposit and dissemination of scientific research documents, whether they are published or not. The documents may come from teaching and research institutions in France or abroad, or from public or private research centers.
L'archive ouverte pluridisciplinaire HAL, est destinée au dépôt et à la diffusion de documents scientifiques de niveau recherche, publiés ou non, émanant des établissements d'enseignement et de recherche français ou étrangers, des laboratoires publics ou privés. 


\title{
LES PROPRIÉTÉS ACOUSTIQUES DES VERRES ISOLANTS A BASSE TEMPÉRATURE
}

\author{
P. DOUSSINEAU \\ Laboratoire d'Ultrasons (*), Université Pierre et Marie-Curie, \\ Tour 13, 4, place Jussieu, 75230 Paris Cedex 05, France
}

\begin{abstract}
Résumé. - Les verres isolants présentent à basse température des propriétés différentes de celles des cristaux. La variation linéaire en température de la chaleur spécifique, la loi en $T^{2}$ de la conductivité thermique, ont conduit à postuler l'existence d'excitations supplémentaires sous la forme de systèmes à deux niveaux d'énergie ayant une densité d'état presque constante. Dans le cadre de ce modèle sont aussi expliqués les deux régimes d'atténuation ultrasonore : résonnante variant comme $T^{-1}$ et de relaxation en $T^{3}$. Le premier régime est sensible à la saturation, ce qui est une autre caractéristique de ces systèmes. Le même modèle explique la variation en $\log T$ de la vitesse de phase. Une fois ces lois expliquées et les coefficients de couplage mesurés, l'utilisation des ondes acoustiques permet l'étude de certaines propriétés des verres. On peut citer l'interaction entre systèmes à deux niveaux, la formation d'échos ultrasonores de spins fictifs. Enfin les propriétés diélectriques anormales à basse température peuvent être étudiées dans des expériences mixtes où interviennent une onde électromagnétique et une onde acoustique.
\end{abstract}

\begin{abstract}
The low temperature properties of amorphous insulators are quite different from those of crystals. In amorphous compounds the linear temperature variation of the specific heat and the $T^{2}$ law of the thermal conductivity led to the postulation of additional excitations in the form of two-level systems with a nearly constant density of states. Using this model the behavior of ultrasonic attenuation can be explained in the two regimes of resonant attenuation (varying as $T^{-1}$ ) and relaxation attenuation (varying as $T^{3}$ ). The first regime is sensitive to saturation which is another feature of these systems. The same model explains the $\log T$ variation of the phase velocity. Having explained these experimental laws and measured the coupling coefficients, further properties of glasses can be studied by the use of acoustic waves, two examples being the interaction between two-level systems and the formation of ultrasonic pseudo-spin echoes. Finally the anomalous dielectric properties at low temperatures can be studied by means of cross-experiments where one electromagnetic wave and one acoustic wave are used simultaneously.
\end{abstract}

1. Introduction. - L'étude des verres au moyen des rayons $\mathbf{X}$ montre que les distances interatomiques sont les mêmes que pour le matériau sous forme cristalline, quand elle existe. Par exemple, pour la silice la distance $\mathrm{Si}-\mathrm{O}$ vaut $1,60 \AA$ comme dans le quartz. Mais à grande distance, les corrélations de position disparaissent : un verre est un milieu désordonné. Considéré à grande échelle, ce désordre, par effet de moyenne, donne au milieu une grande symétrie macroscopique : le verre est isotrope. Pour les propriétés optiques ou élastiques, les verres sont des milieux isotropes comme les liquides.

Donc, au premier abord, un verre apparaît comme un liquide immobilisé par sa viscosité avant d'avoir atteint son équilibre thermodynamique. Et cet état hors d'équilibre peut persister indéfiniment. Dans ces conditions, il est facile de calculer la chaleur spécifique à très basse température $(T<1$ ou $2 \mathrm{~K})$ car elle n'est déterminée que par l'excitation thermique des quanta d'énergie élastique (phonons) de basses fré-

(*) Associé au Centre National de la Recherche Scientifique. quences donc de grandes longueurs d'onde pour lesquels le milieu apparaît continu. C'est la théorie de Debye ; elle donne $C=c_{\mathrm{D}} T^{3}$.

Sans aucun doute possible, l'expérience infirme cette précision. La chaleur spécifique, mesurée sur un grand nombre de verres isolants à basse température, est beaucoup plus grande que prévue et est de la forme $[1,2]$ :

$$
C=c_{1} T+c_{3} T^{3} \text { avec } c_{3}>c_{\mathrm{D}} \text {. }
$$

Tout se passe comme s'il existait des excitations, des degrés de libertés supplémentaires dont l'énergie est de l'ordre de grandeur $10^{-6}$ à $10^{-3} \mathrm{eV}$. En outre, le coefficient $c_{1}$ (dont nous verrons plus loin qu'il est lié à la densité d'états de ces excitations) est à peu près le même pour tous les matériaux amorphes.

Quelles sont ces excitations ? Quelle est leur nature et leur densité spectrale ? L'excès de chaleur spécifique, la limitation du libre parcours moyen des phonons que met en évidence la faible conductibilité thermique, peuvent-ils être attribués aux mêmes excitations ? C'est pour tenter de répondre à ces questions que 
depuis cinq ans environ, les expériences sur les verres se sont multipliées. Si la nature de ces excitations n'est pas encore établie de façon définitive, il fut vite compris qu'elles diffusaient fortement les ondes élastiques. C'est dire que les méthodes acoustiques pouvaient jouer un rôle important dans l'étude de ces matériaux. Dire quel fut ce rôle, quelles furent les informations recueillies et quelles sont les perspectives encore offertes, tel est l'objet du présent exposé.

Après une présentation du modèle utilisé pour décrire les excitations de basses énergies, les résultats de mesures de chaleur spécifique et de conductivité thermique sont rappelés. Ensuite les informations fournies par les études acoustiques (mesures d'atténuation et de variation de vitesse ultrasonore) sont présentées en détail. Enfin un aperçu est donné sur l'utilisation des méthodes acoustiques dans les directions actuelles d'étude des verres.

2. Le modèle. - 2.1 Systèmes A DeUX NiveAuX. Il semble maintenant admis que les propriétés des verres isolants à basse température sont bien décrites par le modèle proposé simultanément par Phillips [3] et Anderson et al. [4]. Ce modèle décrit les excitations supplémentaires de basse énergie, dont les mesures de chaleur spécifique et de conductivité thermique ont révélé l'existence, comme des systèmes à deux niveaux d'énergie $(\mathrm{S} 2 \mathrm{~N})$ dont la densité spectrale $n(2 E)$ serait constante sur une grande gamme d'énergie.

Un tel modèle phénoménologique suffirait pour expliquer la quasi-totalité des expériences faites à ce jour sur les verres isolants sans que la nature de ces systèmes ou défauts soit connue. Cependant, pour fixer les idées, et aussi parce que c'est le plus vraisemblable, on admet généralement que les deux niveaux d'énergie sont les deux états propres d'une particule (atome ou peut-être groupe d'atomes) placée dans un double puits de potentiel et pouvant passer de l'une à l'autre des positions par effet tunnel. Un tel double puits est représenté schématiquement sur la figure 1.

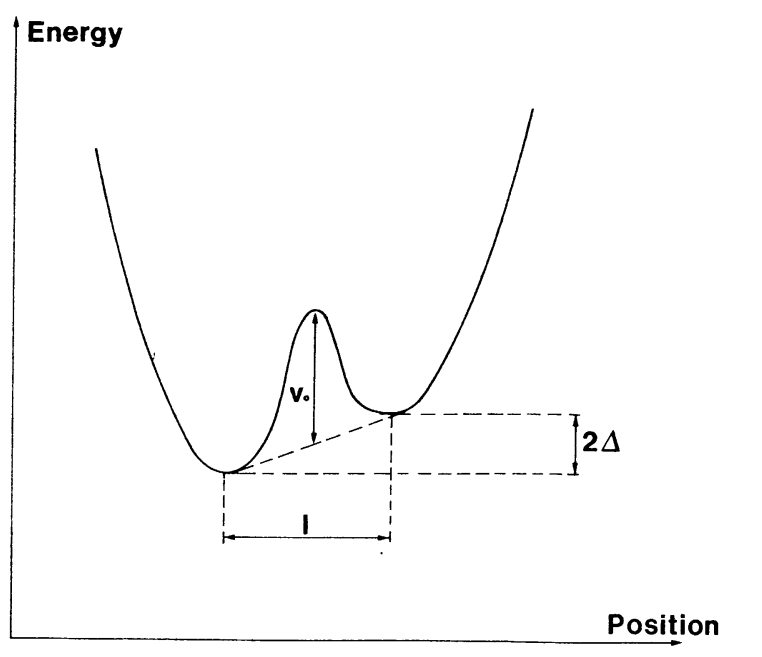

FIG. 1. - Double puits de potentiel avec asymétrie $2 \Delta$, hauteur de la barrière $V_{0}$ et distance $l$ entre les deux minima.
Les états propres ont pour énergie :

$$
E= \pm\left(\Delta^{2}+\Delta_{0}^{2}\right)^{1 / 2}
$$

$\Delta$ où 2 est l'asymétrie du double puits et $\Delta_{0}$ est fonction des caractéristiques de ce puits (hauteur de la barrière de potentiel $V$, distance entre les deux minima $l$ ) et de la masse de la particule. La densité spectrale constante signifie que toutes les valeurs d'énergie $E$ sont également possibles de 0 jusqu'à environ $0,1 \mathrm{eV}$ qui est de l'ordre de l'énergie de fusion $(1000 \mathrm{~K})$.

Il est nécessaire de remarquer qu'une valeur donnée de $E$ peut être obtenue pour différentes combinaisons de valeurs de $\Delta$ et $\Delta_{0}$, c'est-à-dire pour différentes caractéristiques des doubles puits de potentiel. A cause de la structure désordonnée, les valeurs de $\Delta$ et $\Delta_{0}$ varient bien entendu d'un site à l'autre et toute statistique sur l'énergie est en fait une double statistique sur ces deux paramètres.

2.2 SPIN FICTIF. - Il a été proposé de considérer les deux niveaux d'énergie comme étant ceux d'un spin fictif $S=1 / 2$ placé dans un champ magnétique local variant de site en site en intensité comme en orientation [4]. L'avantage de cette description est qu'elle permet l'utilisation du formalisme développé pour le magnétisme.

Ainsi l'hamiltonien du système peut être écrit :

$$
\mathscr{H}_{0}=2 E S_{\mathrm{Z}}
$$

et les deux états propres $\left(S_{Z}= \pm 1 / 2\right)$ ont bien les énergies $\pm E$.

\subsection{COUPLAGE ENTRE UNE DÉFORMATION ÉLASTIQUE} ET LES SYSTÈMES A DEUX NIVEAUX. - Toute déformation de l'environnement d'un défaut modifie les paramètres du double puits et donc les niveaux d'énergie du défaut. Ce mécanisme de couplage avec le réseau est l'analogue de la situation rencontrée en magnétisme et l'hamiltonien de couplage peut s'écrire :

$$
\mathcal{H}_{\mathrm{c}}=\sum_{\alpha \beta} \varepsilon_{\alpha \beta} G_{\alpha \beta, X} S_{X}+\sum_{\alpha \beta} \varepsilon_{\alpha \beta} G_{\alpha \beta, Z} S_{Z}
$$

où $\varepsilon_{\alpha \beta}$ est une composante du tenseur de déformation ( $\alpha \beta$ se réfèrent aux axes géométriques du matériau). $S_{X}$ et $S_{Z}$ sont les composantes du spin fictif. Les axes $X, Y, Z$ varient aléatoirement d'un site à l'autre.

Les termes $\varepsilon_{\alpha \beta} G_{\alpha \beta, X} S_{X}$ qui ne commutent pas avec l'hamiltonien statique $\mathfrak{H}_{0}$ permettent de décrire les transitions résonnantes entre les deux états des $\mathrm{S} 2 \mathrm{~N}$, c'est-à-dire le passage de l'état $\mid-1 / 2>$ d'énergie $-E$ à l'état $\mid+1 / 2>$ d'énergie $+E$ par absorption d'un quantum élastique de fréquence $v$, pourvu que $2 E=h v$ Les autres termes $\varepsilon_{\alpha \beta} G_{\alpha \beta, \mathrm{Z}} S_{\mathrm{Z}}$ représentent la modification de l'énergie des états en présence d'une déformation élastique $\varepsilon_{\alpha \beta}$; ils interviennent dans l'atténuation non résonnante (ou de relaxation) de l'onde élastique.

$G_{\alpha \beta, X}$ et $G_{\alpha \beta, Z}$ sont donc les constantes de couplage entre la déformation élastique et les S $2 \mathrm{~N}$ (ou spinphonon). Elles sont liées aux dérivées $\partial \Delta / \partial \varepsilon_{\alpha \beta}$ et 
$\partial \Delta_{0} / \partial \varepsilon_{\alpha \beta}$. Elles changent bien évidemment d'un spin à l'autre à cause des caractéristiques différentes de chaque double puits de potentiel. Il s'avère donc nécessaire de faire des moyennes sur les $\Delta$ et $\Delta_{0}$. Les valeurs moyennes $\left\langle G_{X}^{2}\right\rangle$ et $\left\langle G_{Z}^{2}\right\rangle$ varient lentement avec $E$ et on suppose généralement qu'elles sont constantes.

3. Propriétés thermodynamiques des verres isolants. - 3.1 Chaleur SPÉCIFIQUe. - Nous nous intéressons uniquement aux basses températures, inférieures à $10 \mathrm{~K}$. La chaleur spécifique a été mesurée par divers groupes dans la gamme comprise entre 0,1 et $10 \mathrm{~K}$ sur de nombreux matériaux de compositions chimiques et de structures différentes. Ceci inclut des verres tels $\mathrm{SiO}_{2}, \mathrm{GeO}_{2}, \mathrm{~B}_{2} \mathrm{O}_{3}, \mathrm{As}_{2} \mathrm{~S}_{3}, 3 \mathrm{SiO}_{2}, \mathrm{Na}_{2} \mathrm{O}$ etc... ainsi que des polymères PMMA, Polystyrène, Lexan ou encore du glycérol ou le vernis GE 7031 [1, 2].

Dans cette région de température et pour tous les amorphes étudiés, les caractéristiques suivantes ont été relevées :

- la chaleur spécifique par unité de volume du matériau non cristallin est toujours beaucoup plus grande que pour le cristal correspondant quand il existe ;

- elle peut se mettre sous la forme

$$
C_{\mathrm{V}}=c_{1} T+c_{3} T^{3} \text {. }
$$

- le coefficient $c_{1}$ a une valeur qui varie très peu (moins de $50 \%$ ) d'un matériau à l'autre. Le coefficient $c_{3}$ est toujours supérieur à la valeur $c_{\mathrm{D}}$ calculée par le modèle de Debye qui est tout à fait correct pour les cristaux à ces températures.

Ce comportement anormal est bien décrit par le modèle des $\mathrm{S} 2 \mathrm{~N}$ : ils sont les excitations supplémentaires qui augmentent la chaleur spécifique. L'effet est analogue à l'anomalie de Schottky produite sur la chaleur spécifique par des vrais spins. Mais la densité d'état constante des $\mathrm{S} 2 \mathrm{~N}$ introduit une différence : au lieu d'une anomalie à une température bien définie, on a une contribution étalée, et plus exactement, linéaire en $T$. On trouve :

$$
C_{\mathrm{v}}=\frac{\pi^{2}}{6} n_{0} k_{\mathrm{B}}^{2} T
$$

La mesure de $C_{\mathrm{v}}$ donne donc la densité d'état $n_{0}(2 E)$ supposée constante. Les écarts à la loi en $T$ peuvent être expliqués par la non-constance de $n(2 E)$. Il faut cependant prendre garde que la chaleur spécifique est sensible à toutes les excitations, y compris celles qui ne sont pas les $S 2 \mathrm{~N}$, en particulier les impuretés $[6,7]$. Moyennant quelques précautions, on a pu obtenir $n_{0} \simeq 2 \times 10^{32} \mathrm{erg}^{-1} \mathrm{~cm}^{-3}$ pour tous les verres.

3.2 CONDUCTIBILITÉ THERMIQUE. - La conductivité thermique des verres isolants a été mesurée dans la même gamme de températures et sur les mêmes matériaux que la chaleur spécifique $[1,2]$. Là aussi, le comportement est différent de celui des cristaux. La conductivité thermique est plus faible et suit approximativement une loi en $T^{2}$ avant d'être constante aux alentours de $10 \mathrm{~K}$ (plateau) puis de croître à nouveau, alors qu'aux mêmes températures, la conductivité thermique d'un cristal parfait est en $T^{3}$. Ces propriétés sont aussi quasiment indépendantes du matériau. Les écarts sont même plus faibles que pour la chaleur spécifique.

Il a été montré que, dans les amorphes, la chaleur était bien transportée par les phonons [8]. Le libre parcours moyen qui en a été déduit $\left(l \simeq 6 \times 10^{-3} \mathrm{~cm}\right.$ à $0,5 \mathrm{~K}$ dans $\mathrm{SiO}_{2}$ ) est plus faible que celui observé dans les cristaux. Toutes ces propriétés ont aussi été expliquées en supposant que, à basse température, les $\mathrm{S} 2 \mathrm{~N}$ agissaient comme diffuseurs supplémentaires pour les phonons. On retrouve alors la loi en $T^{2}$ et la mesure de la valeur absolue de la conductivité thermique donne une valeur pour le produit $n G_{X}^{2}$. La densité d'état étant déjà connue par les mesures de chaleur spécifique, on en déduit le coefficient de couplage $G_{X}$ entre les $\mathrm{S} 2 \mathrm{~N}$ et la déformation. Typiquement $G_{X} \simeq 0,5 \mathrm{eV}$, c'est-à-dire un très fort couplage comparable à celui d'impuretés telles que $\mathrm{OH}^{-}$dans $\mathrm{KCl}$ [9].

A partir de l'ensemble des mesures publiées à ce jour, il semble que la conductivité thermique dans les amorphes ne dépende que très peu de l'état de pureté du matériau. Elle serait donc plus intrinsèque que la chaleur spécifique $[6,7,10]$.

4. Les méthodes acoustiques. - Les mesures de conductivité thermique sont déjà des mesures de type acoustique en ce sens qu'elles renseignent sur le libre parcours moyen des phonons thermiques. Mais ce sont des mesures globales car elles font intervenir simultanément toutes les polarisations d'ondes élastiques et une large gamme de fréquences (telles que $\hbar \omega \sim k T$ ). On voit donc l'intérêt d'expériences plus fines, plus analytiques, où n'interviendraient qu'une seule polarisation et une seule fréquence. C'est là le domaine de l'acoustique.

4.1 Atténuation D'Une onde élastique. - Les mesures d'absorption ultrasonore dans les verres isolants révèlent aussi un comportement spécifique différent de celui des solides cristallisés. Ainsi, les premières mesures, faites à basse température, par des expériences d'effet Brillouin stimulé (donc à forte intensité acoustique) ont donné une valeur de libre parcours moyen bien supérieure à celle déduite des mesures de conductivité thermique [11]. Ce désaccord a pu être expliqué par la suite. Des expériences de propagation ultrasonore ont en effet montré que l'atténuation mesurée dépend de la puissance ultrasonore. Grande à bas niveau, elle diminue lorsque le niveau augmente $[12,13,14,15]$.

Cette saturation de l'absorption ultrasonore est expliquée dans le cadre du modèle des $\mathrm{S} 2 \mathrm{~N}$ qui est 
d'ailleurs le seul à en rendre compte. C'est cette théorie que l'on examine d'abord succinctement.

Puisqu'elle est couplée aux S $2 \mathrm{~N}$, une onde ultrasonore de fréquence $\omega$ peut induire des transitions résonnantes pour ceux dont les deux niveaux d'énergie sont distants de $2 E=\hbar \omega$. Lorsque le taux de ces transitions est trop grand pour que l'équilibre thermique soit rétabli par la relaxation avec les phonons thermiques, les populations des deux niveaux tendent à s'égaliser. L'atténuation ultrasonore devient alors plus faible : c'est la saturation.

Le libre parcours moyen $l_{\mathrm{R}}$ et l'atténuation $l_{\mathrm{R}}^{-1}$ dus aux effets résonnants ont été calculés $[13,5]$. On obtient :

$$
l_{\mathrm{R}}^{-1}=\frac{1}{\left(1+\Phi / \Phi_{\mathrm{c}}\right)^{1 / 2}} \frac{\pi \omega n G_{X}^{2}}{4 \rho v^{3} \hbar} \text { th }\left(\frac{\hbar \omega}{2 k T}\right)
$$

où $\rho$ est la masse volumique du matériau.

$v$ la vitesse de propagation de l'onde acoustique.

$T$ la température.

$k$ la constante de Boltzmann.

$\Phi$ le flux de l'onde acoustique incidente.

$\Phi_{c}$ le flux critique donné par :

$$
\frac{G_{X}^{2} \Phi_{c}}{2 \rho v^{3} \hbar^{2}} T_{1} T_{2}=1
$$

$T_{1}$ et $T_{2}$ étant respectivement les temps de relaxation $\mathrm{S} 2 \mathrm{~N}$-réseau et $\mathrm{S} 2 \mathrm{~N}-\mathrm{S} 2 \mathrm{~N}$.

La formule donnant $l_{\mathrm{R}}^{-1}$ est très intéressante car elle contient des critères directs de la validité du modèle. D'abord, le facteur th $(\hbar \omega / 2 k T)$ est caractéristique de la variation avec la température de la différence de population de systèmes à deux niveaux (spins ou autres). Ensuite, à température donnée, la différence de population de $\mathrm{S} 2 \mathrm{~N}$ à la résonance varie en fonction de l'intensité du champ qui induit les transitions comme

$$
n=n_{0}\left(1+\frac{\Phi}{\Phi_{c}}\right)^{-1}
$$

c'est un résultat bien connu en résonance paramagnétique électronique. Ici, pour une propagation ultrasonore, ce résultat est valable localement :

$$
n(x)=n_{0}\left(1+\frac{\Phi(x)}{\Phi_{c}}\right)^{-1}
$$

$\Phi(x)$ varie au cours de la propagation à cause de l'atténuation de l'onde. Quand on tient compte de cette variation, on trouve

$$
l_{\mathrm{R}}^{-1} \sim\left(1+\frac{\Phi}{\Phi_{\mathrm{c}}}\right)^{-1 / 2}
$$

qui est donc aussi caractéristique de la saturation d'une population de S 2 N.

Le processus résonnant n'est pas le seul mécanisme possible d'absorption ultrasonore. En modifiant l'énergie des S $2 \mathrm{~N}$, l'onde acoustique perturbe leur équilibre thermique. Le retour à l'équilibre s'effectue par un processus de relaxation entre $S 2 \mathrm{~N}$ et phonons $[16,17]$. Le temps caractéristique en est $T_{1}$.

Cette contribution a aussi été calculée et on trouve [16]

$$
l_{\mathrm{N} . \mathrm{R}}^{-1}=\frac{\pi^{3}}{128} \frac{n_{0} G_{Z}^{2}}{\rho^{2} v^{3}} \frac{k^{3} T^{3}}{\hbar^{4}}\left(\frac{G_{X, \mathrm{~L}}^{2}}{v_{\mathrm{L}}^{5}}+\frac{2 G_{X, \mathrm{~L}}^{2}}{v_{X}^{5}}\right) .
$$

Cette formule est valable à basse température, lorsque $\omega T_{1}(\omega, T) \gg 1$. Elle montre que dans ce cas, l'atténuation de relaxation ne dépend pas de la fréquence et qu'elle n'est pas saturable.

Expérimentalement, la dépendance en puissance de l'atténuation ultrasonore a été observée dans différents verres isolants dans la gamme de fréquences s'étendant de 0,5 à $10 \mathrm{GHz}[12,13,14,15,18,19]$. Les caractéristiques principales des résultats sont les mêmes et sont pratiquement indépendantes du matériau étudié. La figure 2 reproduit une courbe d'absorption $l^{-1}$ en fonction de la puissance $P$ à $0,732 \mathrm{GHz}, 0,48 \mathrm{~K}$ dans un borosilicate [15]. A forte puissance, $l^{-1}$ est indépen-

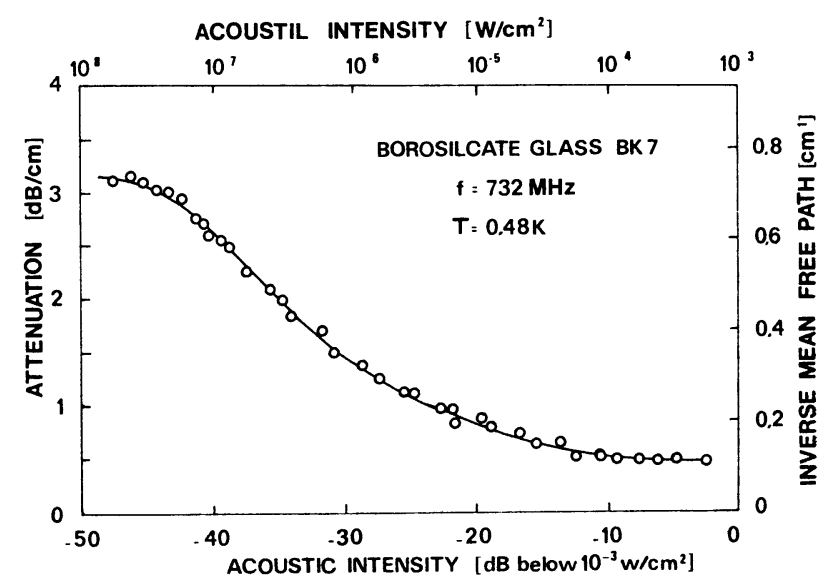

FIG. 2. - Dépendance en intensité de l'atténuation d'ondes acoustiques longitudinales dans un borosilicate BK 7. (W. Arnold et al. J. Non Crystall. Solids. 14 (1974) 192.)

dant de $P$ et est faible ( $\sim 0,1 \mathrm{~cm}^{-1}$ dans ce cas). A basse puissance $l^{-1}$ est aussi indépendant de $P$, mais est grande comparée à l'atténuation précédente $\left(\sim 0,8 \mathrm{~cm}^{-1}\right)$. Dans le domaine intermédiaire $l^{-1}$ varie avec la puissance suivant une loi en $P^{-1 / 2}$ ou $\Phi^{-1 / 2}$. A l'aide du modèle précédent, les deux régions extrêmes sont identifiées comme celles où dominent respectivement l'atténuation non résonnante et l'atténuation résonnante. Dans la région intermédiaire, la théorie prévoit bien une loi en $P^{-1 / 2}$ qui a été vérifiée dans toute la gamme de fréquences explorée à ce jour.

Si l'atténuation est mesurée en fonction de la température, le résultat est différent suivant la puissance ultrasonore dans le cristal. A forte puissance, la contribution non résonnante, prédomine et on observe une dépendance en $T^{3}$ de $l^{-1}$. A basse puissance, la contribution résonnante domine et l'atténuation croît 
lorsque la température diminue avec une loi en $T^{-1}$. La figure 3 reproduit l'ensemble de ces résultats [15]. La dépendance en $\omega^{2}$ attendue dans le cas $\hbar \omega \ll k T$ a aussi été vérifiée.

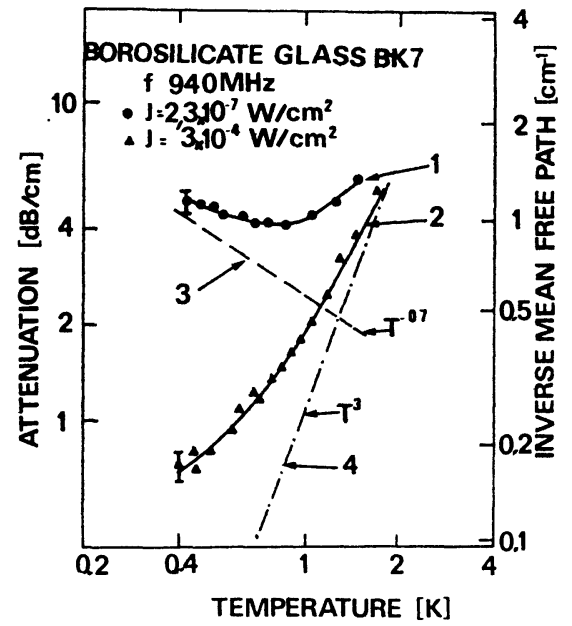

Fig. 3. - Dépendance en température de l'atténuation ultrasonore dans un borosilicate à $940 \mathrm{MHz}$ à deux intensités acoustiques différentes. La courbe 3 est le résultat de la différence entre les courbes 1 et 2 . La courbe 4 est la courbe 2 de laquelle a été retranchée une atténuation résiduelle (ici $0,6 \mathrm{~dB} / \mathrm{cm}$ ) indépendante de la température. (Arnold W. et al. J. Non Crystall. Solids 14 (1974) 192.)

Avec une onde ultrasonore de fréquence $0,592 \mathrm{GHz}$ et dans la région de température entre $20 \mathrm{mK}$ et $0,5 \mathrm{~K}$, la loi en th $(\hbar \omega / 2 k T)$ a pu être mise en évidence comme le montre la figure 4 [20].

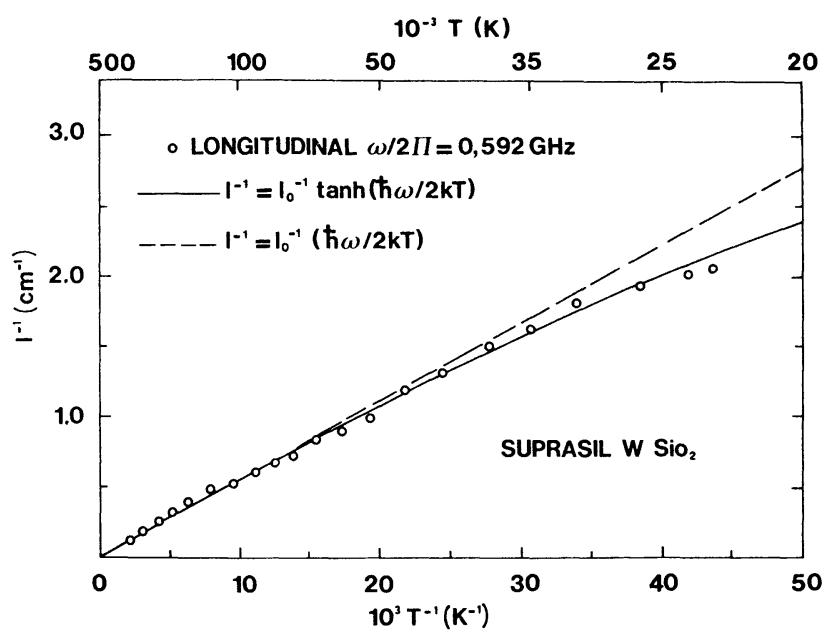

Fig. 4. - Dépendance en température de l'atténuation d'ondes acoustiques longitudinales et transversales dans un verre de silice. (Golding B. et al. Phys. Rev. B 14 (1976) 1660.)

Il est important de noter que ces expériences ont été menées sur plusieurs matériaux incluant des silices de diverses puretés et des borosilicates. Les résultats sont similaires dans tous les cas. Et même plus, de ces mesures, il est possible d'évaluer les constantes de couplage $S 2 \mathrm{~N}$ - déformation élastique $G_{X}$ et $G_{Z}$, ou plus exactement les produits $n G^{2}$; les valeurs déduites sont, à un facteur deux près, les mêmes pour tous les matériaux. La densité d'états $n$ étant connue par les mesures de chaleur spécifique, on peut calculer $G_{X}$ et $G_{Z}$. On trouve $G_{X}$ de l'ordre de $0,5 \mathrm{eV}$ et $G_{Z}$ voisin de $1 \mathrm{eV}$.

4.2 VARIATION DE VITESSE. - A toute atténuation ultrasonore est liée une variation de la vitesse de propagation. Les deux quantités sont liées par les relations de Kramers-Krönig. De l'expression de l'atténuation, il est facile d'obtenir la variation de vitesse.

La contribution résonnante s'écrit dans le cas d'une densité d'états constante et dans l'approximation $\hbar \omega \ll k T[21]:$

$$
\left(\frac{\Delta v}{v}\right)_{R}=\left(\frac{v(T)-v\left(T_{0}\right)}{v\left(T_{0}\right)}\right)_{R}=\frac{n G_{X}^{2}}{4 \rho v^{2}} \log \left(\frac{T}{T_{0}}\right) .
$$

$T_{0}$ est une température de référence choisie arbitrairement. Cette contribution est indépendante de la fréquence et correspond à une augmentation de la vitesse avec la température contrairement à ce qui est généralement observé dans un cristal.

A très basse température $(\hbar \omega \gg k T)$, la variation de vitesse devient indépendante de $T$, mais dépend de $\omega$. Les deux régions se raccordent en faisant apparaître un minimum à $\hbar \omega \simeq 2,2 k T[19,23]$.

La contribution non résonnante peut aussi être calculée. Dans l'approximation des basses températures $\left(\omega T_{1}(T, \omega) \gg 1\right)$, on trouve tous calculs faits

$\left(\frac{\Delta v}{v}\right)_{\mathrm{N} \cdot \mathrm{R}}=-\frac{\pi^{4}}{4 \times 42} \frac{n G_{Z}^{2}}{\rho^{3} v^{2}} \frac{k^{6} T^{6}}{\hbar^{8} \omega^{2}}\left(\frac{G_{X . \mathrm{L}}^{2}}{v_{\mathrm{L}}^{5}}+\frac{2 G_{X . \mathrm{T}}^{2}}{v_{\mathrm{T}}^{5}}\right)^{2}$

où les indices $\mathrm{L}$ et $\mathrm{T}$ se réfèrent aux ondes longitudinales et transversales et où $\mathrm{A}$ est une constante numérique.

La variation de vitesse est cette fois négative.

Comme pour l'atténuation, à basse température la partie résonnante domine, tandis que la non résonnante l'emporte à plus haute température. Les deux contributions étant de signes contraires, la courbe $(\Delta v / v)_{\text {total }}$ en fonction de $T$ passe par un maximum dont la position dépend de la fréquence.

Il faut remarquer que, contrairement à ce qui se passe pour l'atténuation, la contribution résonnante à la variation de vitesse n'est pas saturable. L'explication en est que les $\mathrm{S} 2 \mathrm{~N}$ qui influent sur $\Delta v / v$ mesuré à $\omega$ fixé s'étendent sur une large bande d'énergie (ou de fréquence) de l'ordre de $k T$; au contraire, pour l'atténuation les $\mathrm{S} 2 \mathrm{~N}$ concernés sont uniquement ceux à la fréquence $\omega$ de l'onde acoustique (à la largeur de raie près).

Expérimentalement, la variation de vitesse ultrasonore a été mesurée pour des fréquences allant de 
$30 \mathrm{MHz}$ à $2 \mathrm{GHz}$ dans la gamme de températures comprise entre $20 \mathrm{mK}$ et $5 \mathrm{~K}[21,22,23,24]$.

La figure 5 reproduit les mesures de variation de vitesse à 30,90 et $150 \mathrm{MHz}$ dans une variété de silice entre $0,3 \mathrm{~K}$ et $4,2 \mathrm{~K}$ [21]. On distingue bien les carac-

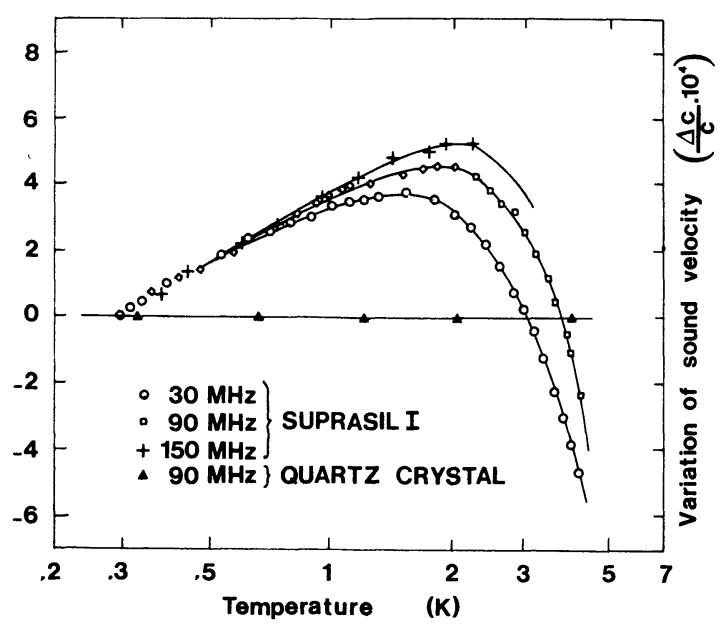

Fig. 5. - Variation relative de la vitesse d'une onde acoustique longitudinale. Les courbes en fonction de la température sont normalisées à la température la plus basse. (Piché L. et al. Phys. Rev. Lett. 32 (1974) 1426.)

téristiques prévues par le modèle des $\mathrm{S} 2 \mathrm{~N}$ : une croissance en $\log T$ indépendante de la fréquence aux plus basses températures, puis après un maximum dépendant de $\omega$, une décroissance rapide. L'indépendance de $\Delta v / v$ en fonction du flux ultrasonore incident a aussi été vérifiée. Ces mesures fournissent une nouvelle évaluation du produit $n G_{X}^{2}$ en bon accord avec la valeûr déduite des mesures d'atténuation et de conductivité thermique.

Des expériences ont aussi été faites à plus haute fréquence (jusqu'à $2 \mathrm{GHz}$ ) et plus basse température (jusqu'à $20 \mathrm{mK}$ ), atteignant ainsi la région $\hbar \omega \gtrsim k T$ [23]. Les résultats en sont reproduits sur la figure 6. Entre $0,1 \mathrm{~K}$ et $1 \mathrm{~K}$, on retrouve bien la loi en $\log T$. A des températures inférieures, la variation de vitesse tend à devenir indépendante de $T$ avec une valeur qui est fonction de $\omega$. A $1,94 \mathrm{GHz}$, on peut observer un minimum. Toutes ces courbes sont en bon accord avec les expressions calculées numériquement. Il est néanmoins nécessaire pour obtenir un meilleur accord de supposer une petite variation de la densité d'états avec l'énergie, ce qui est déjà le cas pour les résultats de chaleur spécifique [2].

Des expériences de diffusion Brillouin spontanée ont permis de mesurer la variation de vitesse d'ondes acoustiques de fréquence $33 \mathrm{GHz}$ entre $1,5 \mathrm{~K}$ et $20 \mathrm{~K}$ dans de la silice [24]. Le même type de comportement a été observé, ce qui est une extension vers les hautes fréquences de la validité du modèle des $\mathrm{S} 2 \mathrm{~N}$.

Enfin, il faut noter que la variation de vitesse ultrasonore présente le même caractère d'universalité que les

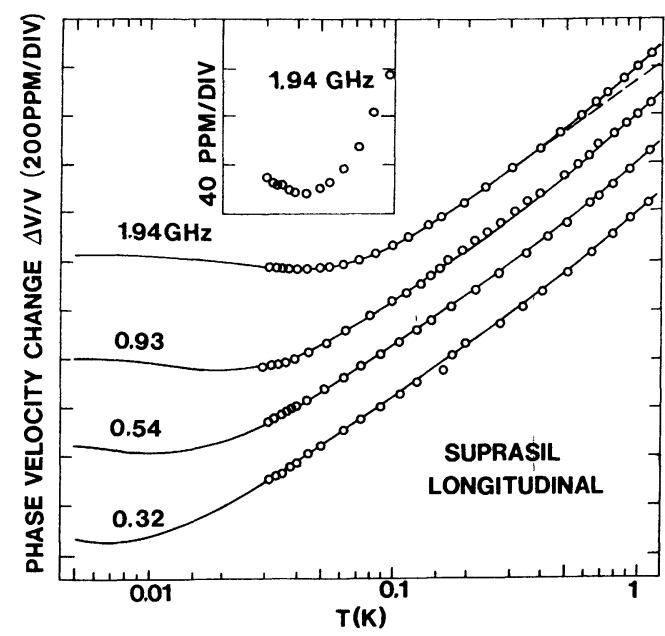

FIG. 6. - Variation relative de la vitesse d'ondes acoustiques longitudinales dans un verre de silice en fonction de la température et de la fréquence. Chaque courbe est décalée arbitrairement de $2 \times 10^{-4}$ à $1 \mathrm{~K}$. La ligne continue représente la vitesse calculée avec une correction quadratique en énergie pour la densité d'états. La courbe en traits interrompus montre la variation de vitesse pour une densité d'états constante. (Golding B. et al. Phys. Rev. Lett. 37 (1976) 1248.)

mesures d'atténuation, de conductivité thermique et dans une moindre mesure, de chaleur spécifique.

Pour résumer ce paragraphe, on peut dire que les expériences d'atténuation acoustique permettent des tests simples, directs (et bien vérifiés) du modèle des systèmes à deux niveaux. Mais les mesures sont délicates car la nécessité d'obtenir une désaturation complète impose d'opérer à des niveaux ultrasonores extrêmement bas ; et plus la fréquence est élevée, plus la difficulté est grande. Les mesures de dispersion acoustique, au contraire, sont plus aisées car l'intensité y est sans influence. Mais, leur interprétation est moins directe car la dispersion fait intervenir les propriétés des $\mathrm{S} 2 \mathrm{~N}$ de toute fréquence (relation de KramersKrönig).

Les deux types d'expériences permettent la mesure de constantes de couplage que l'on peut mettre sous la forme sans dimension

$$
C=\frac{n_{0} G_{X}^{2}}{4 \rho v^{2}} \simeq 5 \times 10^{-4}
$$

qui dépendent peu du matériau.

5. Développements actuels des méthodes acoustiques. - Les expériences de propagation ultrasonore (mesures d'atténuation et de variation de vitesse) ont fait largement progresser la connaissance des verres isolants à basse température.

Actuellement, l'utilisation des ondes acoustiques s'oriente dans deux directions principales : d'une part l'étude des interactions des S $2 \mathrm{~N}$ entre eux et avec le réseau, d'autre part les expériences mixtes où est étudiée l'influence mutuelle d'une onde électromagnétique et d'une onde acoustique dans un verre. 
5.1 INTERACTION DES S $2 \mathrm{~N}$ ENTRE EUX ET AVEC LE RÉSEAU. - Les expériences acoustiques ont montré le fort couplage $\mathrm{S} 2 \mathrm{~N}$-déformation élastique ainsi qu'en témoignent les valeurs de $G_{X}$ et $G_{Z}$. Le problème des interactions entre S $2 \mathrm{~N}$ n'a été abordé que plus tard. Il a été proposé que l'échange de phonons virtuels pouvait être un mécanisme de couplage efficace [5].

Le temps de relaxation $T_{2}$ caractéristique des interactions entre défauts a été mesuré d'abord par une technique utilisant deux ondes acoustiques. La première onde sature les $\mathrm{S} 2 \mathrm{~N}$ qui ont la même fréquence qu'elle et aussi les $\mathrm{S} 2 \mathrm{~N}$ de fréquences voisines à cause des interactions entre $\mathrm{S} 2 \mathrm{~N}$. Si une autre onde de fréquence assez proche se propage simultanément dans l'échantillon, elle interagit avec une population modifiée et son atténuation et sa vitesse sont donc fonction des caractéristiques de la première onde et de tous les processus de relaxation $[26,27]$.

La largeur de raie (reliée à $T_{2}$ ) ainsi mesurée est dépendante des puissances des deux ondes. Une étude systématique en fonction des deux puissances a permis d'atteindre la largeur de raie intrinsèque $\Gamma_{2}$. A $1,5 \mathrm{~K}$, on trouve $\Gamma_{2} \sim 40 \mathrm{MHz}$, soit $T_{2} \sim 5 \times 10^{-9} \mathrm{~s}$ [27].

Le temps de relaxation $\mathrm{S} 2 \mathrm{~N}$-réseau $T_{1}$ a été obtenu par une technique similaire [19]. Des mesures complémentaires sont nécessaires.

Il manque encore une exploration de $T_{2}$ et $T_{1}$ dans une large gamme de températures.

Une technique plus prometteuse semble être celle dite des échos de spins fictifs [28], le formalisme pour la décrire est celui utilisé pour les échos de (vrais) spins, ou mieux, les échos ultrasonores de (vrais) spins [29]. Si deux impulsions ultrasonores de faible largeur $(\Delta t \sim 100 \mathrm{~ns})$ séparées par un temps $\tau$ se propagent successivement dans un verre isolant, il apparaît au temps $2 \tau$ un écho ultrasonore dont le temps de décroissance (en fonction de $\tau$ ) est $T_{2}$ (voir Fig. 7). Si une

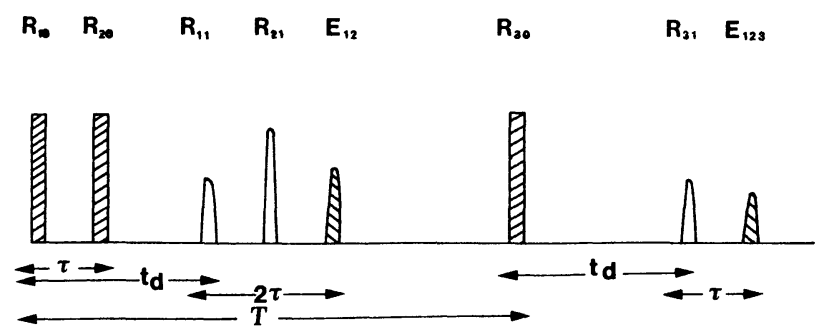

FIG. 7. - Représentation schématique d'une expérience d'échos. $R_{10}, R_{20}$ et $R_{30}$ sont les trois impulsions électromagnétiques émises aux instants $0, \tau$ et $\mathrm{T}$ respectivement. $R_{11}, R_{21}, R_{31}$ sont les trois échos ultrasonores détectés ( $t_{\mathrm{d}}$ est le temps de parcours ultrasonore dans l'échantillon. $E_{12}$ et $E_{123}$ sont les échos de spins fictifs à $2 \tau$ et $T+\tau$. Il n'a été représenté qu'un seul train d'ondes ultrasonores afin de simplifier.

troisième impulsion ultrasonore est engendrée à un instant $T$ après la première, un écho ultrasonore au temps. $T+\tau$ est détecté. Le temps caractéristique de décroissance de cet écho (en fonction de $T$ ) est $T_{1}$.
A $20 \mathrm{mK}$ et $0,68 \mathrm{GHz}$; les valeurs mesurées sont $T_{2}=14 \mu \mathrm{s}, T_{1}=200 \mu \mathrm{s}$ [28]. La valeur numérique de $T_{1}$ peut être calculée à partir des valeurs obtenues pour $G_{X}$ dans une expérience ultrasonore ; elle est en très bon accord avec le $T_{1}$ mesuré, ce qui montre la cohérence de l'ensemble de ces expériences. La variation de $T_{2}$ et $T_{1}$ en fonction de la température n'a pas été encore étudiée, non plus que l'effet d'un éventuel goulot d'étranglement des phonons sur $T_{1}$.

L'étude de la relaxation des $\mathrm{S} 2 \mathrm{~N}$ n'en est qu'à ses débuts. Les expériences ont jusqu'ici un caractère exploratoire. La voie à suivre est d'une part d'élargir la gamme de températures, d'autre part d'étendre les mesures à des matériaux différents.

\subsection{EXPÉRIENCES MIXTES ÉLECTRIQUES-ÉLASTIQUES. -} Ces expériences font suite à la découverte de la variation anormale de la constante diélectrique dans les verres isolants dans la région de température allant de 0,5 à $5 \mathrm{~K}$. La variation relative $\Delta \varepsilon / \varepsilon$ qui est reliée à celle de la vitesse $\mathrm{c}$ des ondes électromagnétiques par : $\Delta \varepsilon / \varepsilon=-2 \Delta c / c$ donne pour cette dernière une loi en Log $T$ suivi d'une décroissance [30].

Comme pour la variation de vitesse ultrasonore à laquelle il est trictement analogue, ce comportement en fonction de la température est dû à l'interaction avec des systèmes à deux niveaux à densité d'état constante sur une large bande d'énergie. Contrairement aux propriétés acoustiques, les propriétés diélectriques des verres isolants dépendent du matériau étudié et plus précisément de son état de pureté [31].

Il a alors été imaginé des expériences mixtes où est étudiée l'influence d'une onde électromagnétique irradiant le matériau sur l'atténuation d'une onde acoustique de fréquence voisine se propageant dans le même verre [32, 33]. Dans l'expérience inverse, on mesure la modification de l'impédance d'une cavité hyperfréquence dans laquelle est placé un échantillon lorsqu'une onde ultrasonore s'y propage [33]. Les expériences ont montré que l'influence mutuelle des deux types d'ondes est importante et se fait par l'intermédiaire des mêmes S 2 N. L'existence de cet effet prouve que des systèmes à deux niveaux sont couplés à la fois à l'onde électrique et à l'onde élastique. L'extension de ces expériences à divers matériaux amorphes de pureté variable doit pouvoir renseigner sur l'origine microscopique des systèmes à deux niveaux.

6. Conclusion. - Les propriétés physiques des verres isolants à basse température sont différentes de celles des matériaux cristallisés comme l'ont d'abord mis en évidence les expériences de chaleur spécifique et de conductivité thermique. Le modèle des systèmes à deux niveaux rend compte de ces propriétés et prévoit des propriétés acoustiques anormales. L'observation expérimentale de la saturation de l'atténuation ultrasonore, de deux contributions différentes (résonnante et non résonnante) à cette atténuation ainsi qu'à la variation de vitesse des ondes élastiques a fourni parmi 
les meilleures confirmations de ce modèle. Ces expériences ont aussi permis d'étudier quelques propriétés des $\mathrm{S} 2 \mathrm{~N}$. Ainsi, il a été montré que les constantes de couplage $G_{X}$ et $G_{Z}$ qui gouvernent l'ensemble des propriétés acoustiques ont une valeur quasi universelle, indépendante de la nature du matériau.

Disposant d'un modèle dont la validité ne semble plus devoir être mise en doute, on peut maintenant envisager l'étude des propriétés des $\mathrm{S} 2 \mathrm{~N}$ en particulier les temps de relaxation $\mathrm{S} 2 \mathrm{~N}-\mathrm{S} 2 \mathrm{~N}$ et $\mathrm{S} 2 \mathrm{~N}$-réseau déjà abordés.

Il est permis aussi d'imaginer un certain nombre de développements à partir du modèle des S 2 N. Ainsi, des mesures de dilatation à basse température ont mon- tré que la valeur de la constante de Gruneisen est particulièrement grande [34], ce qui est expliqué par le modèle des S 2 N [35]. Ceci indique que les anharmonicités élastiques doivent être importantes dans les verres. C'est tout un domaine d'étude qui est encore à explorer.

Un problème majeur subsiste pour la connaissance des verres à basse température : l'origine microscopique de ces systèmes à deux niveaux. Par l'intermédiaire des expériences mixtes électrique-élastique, l'acoustique doit pouvoir donner d'intéressants renseignements.

Je remercie A. Levelut pour toutes les critiques et suggestions faites au cours de la rédaction de cet article.

\section{Bibliographie}

[1] Zeller, R. C., Pohl, R. O., Phys. Rev. B 4 (1971) 2029.

[2] Stephens, R. B., Phys. Rev. B 8 (1973) 2896.

[3] Phillips, W. A., J. Low Temp. Phys. 7 (1972) 351.

[4] Anderson, P. W., Halperin, B. I., Varma, C. M., Phil. Mag. 25 (1972) 1.

[5] Joffrin, J., Levelut, A., J. Physique 36 (1975) 811.

[6] Lasjaunias, J. C., RaveX, A., VandorPe, M., HunklinGER, S., Solid State Commun. 17 (1975) 1045.

[7] Stephens, R. B., Phys. Rev. B 13 (1976) 852.

[8] Zaitlin, M. P., Anderson, A. C., Phys. Rev. Lett. 33 (1974) 1158 ; Phys. Rev. B 12 (1975) 4475.

[9] Narayanamurti, V., Pohl, R. O., Rev. Mod. Phys. 42 (1970) 201.

[10] Lasjaunias, J. C., Thoulouze, D., Pernot, F., Solid State Commun. 14 (1974) 957.

[11] Heinicke, W., Winterling, G. Dransfeld, K., J. Acoust. Soc. Am. 49 (1971) 954.

[12] Hunklinger, S., Arnold, W., Stein, S., Nava, R., Dransfeld, K., Phys. Lett. 42A (1972) 253.

[13] Golding, B., Graebner, J. E., Halperin, B. I., Schutz, R. J., Phys. Rev. Let. 30 (1973) 223.

[14] Hunklinger, S., Arnold, W., Stein, S., Phys. Lett. 45A (1973) 311.

[15] Arnold, W., Hunklinger, S., Stein, S., Dransfeld, K., J. Non Crystall. Solids 14 (1974) 192.

[16] JÄCKLE, J., Z. Phys. 257 (1972) 212.

[17] Jäckle, J., Piché, L., Arnold, W., Hunklinger, S., $J$. Non Crystall. Solids. 20 (1976) 365.

[18] BACHELLERIE, Satellite Symposium of the 8th International Congress on Acoustics. Microwave Acoustics. Lancaster 1974. (E. R. DobBs, J. K. WIGMORE, Lancaster University).
[19] Hunklinger, S., ARnold, W., in Physical Acoustics. (ed. par R. N. Thurston et W. P. Mason. Academic Press) 1976. Vol. 12, p. 155.

[20] Golding, B., Graebner, J. E., Schutz, R. J., Phys. Rev. B 14 (1976) 1660.

[21] Piché, L., Maynard, R., Hunklinger, S., JÄckle, J., Phys. Rev. Lett. 32 (1974) 1426.

[22] Hunklinger, S., Piché, L., Solid State Commun. 17 (1975) 1189.

[23] Golding, B., Graebner, J. E., Kane, A. B., Phys. Rev. Lett. 37 (1976) 1248.

[24] Laermans, C., Piché, L., Arnold, W., Hunklinger, S., à paraître.

[25] Pelous, J., Vacher, R., Solid State Commun. 19 (1976) 627.

[26] Arnold, W., Hunklinger, S., Solid State Commun. 17 (1975) 883.

[27] Bachellerie, A., Doussineau, P., Levelut, A., Ta Thu THUY. J. Physique 38 (1977) 69.

[28] Golding, B., Graebner, J. E., Phys. Rev. Lett. 37 (1976) 852.

[29] Shiren, N. S., Kazyaka, T. G., Phys. Rev. Lett. 28 (1972) 1304.

[30] Schickfus, M. V., Hunklinger, S., Piché, L., Phys. Rev. Lett. 35 (1975) 876.

[31] Schickfus, M. V., Hunklinger, S., J. Phys. C 9 (1976) L-439.

[32] Schickfus, M. V., Laermans, C., Arnold, W., HunklinGER, S. A paraître.

[33] Doussineau, P., Levelut, A., Ta Thu Thuy. J. Physique Lett. 38 (1977) L-37.

[34] White, G. K., Phys. Rev. Lett. 34 (1975) 304.

[35] Phillips, W. A., J. Low Temp. Phys. 11 (1973) 757. 